\title{
Guillain-Barre syndrome and mood disorders
}

\author{
Evangelos Neroutsos ${ }^{1 *}$, Giorgos Vagionis ${ }^{1}$, Markella Fiste ${ }^{2}$ \\ From $1^{\text {st }}$ International Congress on Neurobiology and Clinical Psychopharmacology \\ and European Psychiatric Association Conference on Treatment Guidance \\ Thessaloniki, Greece. 19-22 November 2009
}

\section{Background}

Guillain-Barre syndrome (GBS) is an acute, autoimmune polyradiculoneuropathy affecting the peripheral nervous system, usually triggered by an acute infectious process. It is included in the wider group of peripheral neuropathies. There are several types of GBS, but unless otherwise stated, GBS refers to the most common form, acute inflammatory demyelinating polyneuropathy (AIDP). Clinical hallmarks of this syndrome include symmetric progressive flaccid muscle paresis, areflexia, ataxia, dysautonomia, and respiratory insufficiency in the presence of an increased cerebrospinal fluid protein content, as well as electromyography studies demonstrating evolving demyelination.

\section{Materials and methods}

We report a 20 years old female that after the permanence in the ICU presented to us with a depressed mood. In the progress of the ill from the state of anxiety (especially evident at the initial phase of the disease during the dissemination and maximum intensity of paralysis) she went to a clear presentation of depressive symptoms during the phase of remission. After few days in the neurology clinic she met the criteria of a major depressive episode that was treated with mirtazapine $15 \mathrm{mg}$ daily.

\section{Results}

There were mental status changes in $31 \%$ of GBS patients and in $16 \%$ of controls [1]. Vivid dreams (19\%), illusions (30\%), hallucinations (60\%, mainly visual) and delusions (70\%, mostly paranoid) were included. They appeared a median 9 days after disease onset (range 1-40 days, during the progression or the plateau of the disease), and last a median 8 days. Seven (16\%) patients experienced the symptoms before their admission to the
ICU. Hallucinations were frequently hypnagogic, occurring as soon as the patients closed their eyes. In an older publication [2] anxiety (82\%), acute stress disorder, depressive episodes (67\%) and brief reactive psychosis (25\%) were observed.

\section{Conclusions}

In GBS not only severe psychosis may occur, which may go unrecognised due to the severity of the neurological motor deficits, but also fatigue and depressive episodes as major restrictions of quality of life after the acute phase of GBS. Those are probably the major debilitating factors in chronic inflammatory neuropathies. Symptomatic treatment remains largely empirical and more studies are necessary.

\section{Author details}

${ }^{1}$ General Hospital of Elefsina Thriasio, Elefsina, Athens, Greece. ${ }^{2}$ Psychiatric Hospital of Athens Dromokaitio, Athens, Greece.

Published: 22 April 2010

\section{References}

1. Cochen $V$, et al: Vivid dreams, hallucinations, psychosis and REM sleep in Guillain-Barré syndrome. Brain 2005, 128(Pt 11):2535-45, Epub 2005 Jul 6.

2. Weiss $H$, Rastan V, Móllges W: Psychotic symptoms and emotional distress in patients with Guillain-Barrı syndrome. Eur Neurol 2002, 47(2):74-8.

3. Chan A, Gold R: Neuropsychological/-psychiatric deficits in immunemediated neuropathies. J Neurol 2007, 254(Suppl 2):|193-5.

doi:10.1186/1744-859X-9-S1-S204

Cite this article as: Neroutsos et al:: Guillain-Barre syndrome and mood disorders. Annals of General Psychiatry 2010 9(Suppl 1):S204.

${ }^{1}$ General Hospital of Elefsina Thriasio, Elefsina, Athens, Greece 